\title{
The Phoenix galaxy: UGC 4203 re-birth from its ashes?
}

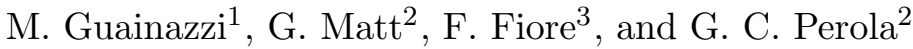 \\ 1 XMM-Newton Science Operation Center, VILSPA, ESA, Apartado 50727, 28080 Madrid, Spain \\ 2 Dipartimento di Fisica, Università degli Studi Roma Tre Via della Vasca Navale 84, 00146 Roma, Italy \\ ${ }^{3}$ Osservatorio Astronomico di Roma, Via dell'Osservatorio, 00144 Monteporzio Catone, Italy
}

Received 3 September 2001 / Accepted 26 March 2002

\begin{abstract}
We report on a dramatic transition between a Compton-thick, reflection-dominated state and a Compton-thin state in the Seyfert 2 galaxy UGC 4203, discovered by comparing a recent (May 2001) XMMNewton observation with ASCA observations performed about six years earlier. This transition can be explained either as a change in the column density of the absorber, maybe due to moving clouds in a clumpy torus, or as the revival of a transient active nucleus, which was in a phase of very low activity when observed by ASCA. If the latter explanation is correct, spectral transitions of this kind provide observational support to the idea that Compton-thick and Compton-thin regions coexist in the same source, the former likely to be identified with the "torus", the latter with dust lanes on much larger scales.
\end{abstract}

Key words. X-rays: galaxies - galaxies: active - galaxies: Seyfert - galaxies: individual: UGC 4203 galaxies: nuclei

\section{Introduction}

X-ray spectra of Seyfert 2 galaxies are always significantly absorbed by line-of-sight cold matter (Awaki et al. 1991; Turner et al. 1997; Risaliti et al. 2001). The X-ray absorber is usually associated with the "torus", responsible - in Seyfert unification models (Antonucci \& Miller 1985; Antonucci 1993) - for the occultation of the Broad Line Regions (BLR).

This scenario may be oversimplified. Keel proposed as early as 1980 that the deficiency of nearly edge-on Seyfert 1 galaxies may be due to dust obscuration, associated with the host galaxy. Maiolino \& Rieke (1995) suggested that intermediate Seyferts are seen through a 100 pc-scale, Compton-thin absorber coplanar with the disk of the host galaxy, whereas only "strict" Seyfert $2 \mathrm{~s}$ are obscured by the inner torus. Expanding this line of thought, Matt (2000) has recently proposed an extension of the unification models, where Compton-thick Seyfert $2 \mathrm{~s}\left(N_{\mathrm{H}} \gtrsim 10^{24} \mathrm{~cm}^{-2}\right)$ are seen through compact, thick matter with a large covering fraction (to be identified with the "torus"), a few tens of parsecs at most far from the nucleus, whereas Compton-thin Seyfert 2s $\left(N_{\mathrm{H}} \lesssim 10^{24} \mathrm{~cm}^{-2}\right)$ are absorbed by matter located at larger distances. The discovery, with HST high-resolution images, that dust lanes on scales from tens to hundred parsecs are common in nearby Seyferts suggests a possible "optical" counterpart for the Compton-thin, X-ray absorbing matter (Malkan et al. 1998; see also

Send offprint requests to: M. Guainazzi,

e-mail: mguainaz@xmm.vilspa.esa.es
Antonucci \& Barvainis 1990 for similar, earlier results on narrow line radio galaxies). Later on, Guainazzi et al. (2001) studied the correlation between the morphology of the circumnuclear dust and the properties of X-ray absorption. Their study indicate that Compton-thin objects prefer dust-rich nuclear environments, whereas the presence of Compton-thick material does not appear to be correlated with the overall dust content.

Such studies have been hampered so far by the limited number of Seyfert 2 galaxies for which good quality $\mathrm{X}$-ray spectra are available. A program has been therefore started to observe with the X-ray satellite XMM-Newton (Jansen et al. 2001) a large sample of Seyfert 2s, selected according to their nuclear dust morphology. We present in this paper the results of the observation of the edgeon maser galaxy (Braatz et al. 1994) UGC 4203 (a.k.a. Mrk 1210; $z=0.013$ ), one of the Seyfert 2s which exhibit broad lines in polarized light (Tran 1995). This observation reveals an unusual transition between a Comptonthick and -thin state, when compared with ASCA observations performed almost six years earlier.

\section{Observations and data reduction}

XMM-Newton observed UGC 4203 on May 52001. The CCD imaging EPIC cameras (Turner et al. 2001; Strüder et al. 2001) were operated in Full Frame mode, with the THIN optical filter. UGC 4203 was too faint to be detected by the high resolution spectroscopy cameras, whose data will not be discussed in this paper. Data were reduced with SAS v5.1 (Jansen et al. 2001), using the most updated version of the calibration files available at 
August 2001. This ensures spectral fitting residuals within $\pm 10 \%$ for all EPIC cameras, accuracy of the absolute position reconstruction within $4^{\prime \prime}$, and of the gain reconstruction $\leq 25 \mathrm{eV}$ at $6 \mathrm{keV}$. The observation of UGC 4203 was affected by a rather high radiation level. The p-n was flooded with background events, and spent most of the observing time in "counting mode". In this mode any information about the position or energy of the incoming photons is lost. For this reason, only MOS data will be discussed in this paper. After data screening, the exposure time is about $7.7 \mathrm{ks}$.

In this paper: errors refer to the $90 \%$ confidence level for one interesting parameter; energies are quoted in the source rest frame, unless otherwise specified.

\section{Spectral analysis}

\subsection{The XMM-Newton observation}

In the MOS cameras, a strong source (MOS 2 count rate in the $0.3-10 \mathrm{keV}$ band of $\left.0.220 \pm 0.006 \mathrm{~s}^{-1}\right)$ is detected. Its centroid position $\left(\alpha_{2000}=08^{\mathrm{h}} 04^{\mathrm{m}} 05.8 ; \delta_{2000}=\right.$ $+05^{\mathrm{h}} 06^{\mathrm{m}} 50^{\mathrm{s}}$ ) coincides within $2^{\prime \prime}$ with the optical nucleus of UGC 4203. The MOS image does not show any evidence of extended emission beyond the instrumental PSF ( 6 " correspond to $100 \mathrm{kpc}$ at the UGC 4203 distance). No flux variation is observed during the observation. We will therefore focus on the time-averaged spectrum only in this paper. X-ray events corresponding to patterns 0 to 12 were used. Source spectra were extracted from a circular region around the image centroid, with a radius of $22^{\prime \prime}$. Spectra were rebinned in order to have at least 50 counts for each spectral bin, to allow a proper use of the $\chi^{2}$ test, and to oversample the instrumental energy resolution by a factor not higher than 3 . Background spectra were extracted from several regions of the central chip, and merged together to increase statistics, once verified that the results were not significantly dependent on the chosen extraction region. A fit to the MOS spectra with a simple, absorbed power-law in the $3-10 \mathrm{keV}$ energy range is acceptable $\left(\chi^{2}=57.0 / 52\right.$ d.o.f.). Excess counts at an observed energy of about $6.3 \mathrm{keV}$ can be interpreted as an emission line, as explained in more details later in this section. The photoelectric column density is $N_{\mathrm{H}}=1.8 \pm_{0.3}^{0.4} \times 10^{23} \mathrm{~cm}^{-2}$, whereas the spectral index, albeit only loosely constrained, is not dissimilar from that typically observed in Seyfert $2 \mathrm{~s}\left(\Gamma=1.6 \pm_{0.4}^{0.3}\right)$.

The extrapolation of the best-fit model in the $3-10 \mathrm{keV}$ band lays, however, well below the data (see Fig. 1). We have therefore added to the hard X-ray absorbed power-law a second unabsorbed power-law, to approximately describe the scattering of the primary nuclear emission by a "warm mirror". Photoelectric absorption with a column density equal to that of our own Galaxy $\left(N_{\mathrm{H}, \mathrm{Gal}}=3.7 \times 10^{20} \mathrm{~cm}^{-2}\right.$; Dickey \& Lockman 1990) has been added to all models hereinafter. If self-absorption effects are neglected, the scattered soft component has the same spectral index of the primary emission; the data do not actually require different indices. The scattering frac-

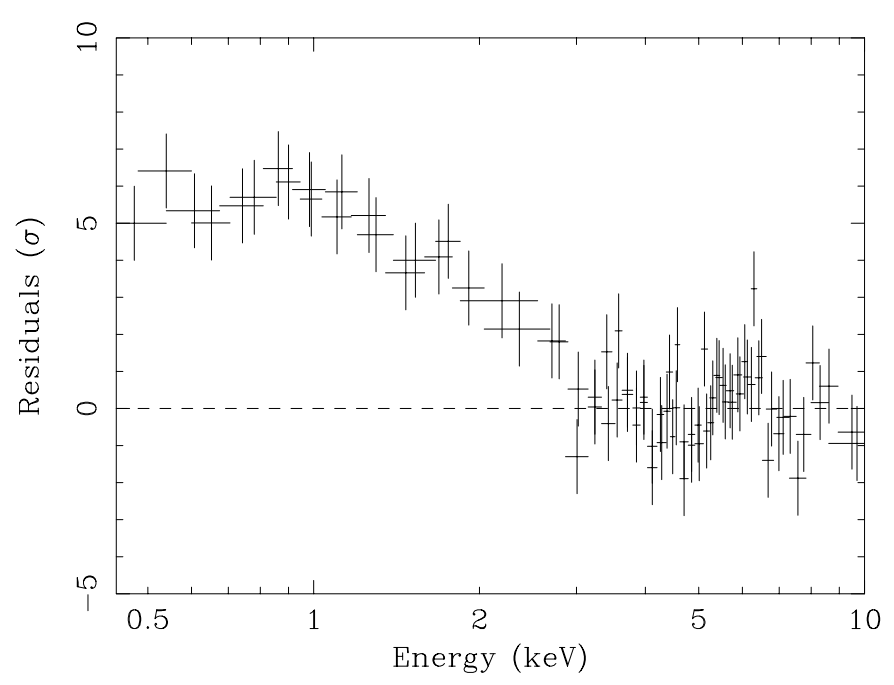

Fig. 1. Residuals in units of standard deviations when the best-fit photoelectric absorbed power-law model, applied in the 3-10 keV band, is extrapolated redwards.

tion is $1.3 \pm_{0.7}^{0.3} \%$. Other descriptions of the soft excess, like e.g. thermal emission from an optically thin collisionally ionized plasma which may be produced in regions of intense star formation, are, however, also consistent with the data. The results on the hard X-ray spectrum are not significantly dependent on the soft excess modeling.

The best-fit parameters and results are summarized in Table 1. Three narrow (i.e.: intrinsic width $\sigma=0$ ) emission lines are also required to get an acceptable fit $\left(\chi^{2}=65.1 / 70\right.$ d.o.f. $)$. One of them $\left(E_{\mathrm{c}} \simeq 6.41 \mathrm{keV}\right.$, $\Delta \chi^{2}=14.1$, corresponding to a confidence level $\left.\simeq 99.9 \%\right)$ is consistent with $\mathrm{K}_{\alpha}$ fluorescent emission from neutral iron. The other two lines have $E_{\mathrm{c}} \simeq 0.93 \mathrm{keV}$ and $E_{\mathrm{c}} \simeq 1.79 \mathrm{keV}$, respectively. The corresponding improvement in the quality of the fit is $\Delta \chi^{2}=14.1$ and $\Delta \chi^{2}=$ 4.2 , respectively, the latter corresponding to a confidence level of $88.8 \%$ only. Their centroid energies are consistent with $\mathrm{K}_{\alpha}$ fluorescent transitions of $\mathrm{H}$-like neon and silicon, probably due to radiative recombination and resonant scattering in a photoionized plasma (Bianchi et al. 2000; Sako et al. 2000; Sambruna et al. 2001). All the detected lines are unresolved, even if the upper limits to their widths are very large. Spectrum and residuals for the bestfit model are shown in Fig. 2.

The $0.5-2.0 \mathrm{keV}(2.0-10 \mathrm{keV})$ flux measured by XMM-Newton is $2.6 \times 10^{-13}$ erg $\mathrm{cm}^{-2} \mathrm{~s}^{-1}(9.7 \times$

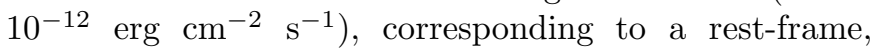
absorption-corrected $2-10 \mathrm{keV}$ luminosity of $1.9 \times$ $10^{43} \mathrm{erg} \mathrm{s}^{-1}$ for the transmitted nuclear emission.

\subsection{ASCA observation}

ASCA observed UGC 4203 twice during autumn 1995, approximately three weeks apart (October 18 and November 12). An analysis of the ASCA data is presented by Awaki et al. (2000). We have combined the two observations and reanalyzed the data, obtaining basically their same results. The hard X-ray spectrum 
Table 1. Best-fit parameters and results of the XMM-Newton observation of UGC 4203. Model scenarios are explained in text.

\begin{tabular}{cccccc}
\hline \hline$\Gamma$ & $\begin{array}{c}N_{\mathrm{H}} \\
\left(10^{23} \mathrm{~cm}^{-2}\right)\end{array}$ & $\begin{array}{c}E_{\mathrm{c}} \\
(\mathrm{keV})\end{array}$ & $\begin{array}{c}I_{\mathrm{c}} \\
\left(10^{-5} \mathrm{~cm}^{-2} \mathrm{~s}^{-1}\right)\end{array}$ & $\begin{array}{c}E W \\
(\mathrm{eV})\end{array}$ & $\chi^{2} /$ d.o.f. \\
\hline $2.0 \pm 0.2$ & $2.14 \pm_{0.16}^{0.20}$ & $0.93 \pm_{0.05}^{0.02}$ & $1.7 \pm_{0.9}^{0.4}$ & $130^{\dagger}$ & $65.1 / 70$ \\
& & $1.79 \pm_{0.09}^{0.06}$ & $4 \pm_{3}^{4}$ & $110^{\dagger}$ & \\
& & $2.9 \pm_{1.3}^{2.3}$ & $130^{\ddagger}$ & \\
\hline
\end{tabular}

$\dagger$ Against the scattered continuum; ${ }^{\ddagger}$ against the primary continuum.

Table 2. Best-fit parameters and results of the ASCA observation of UGC 4203. Model scenarios are explained in text. $N_{\mathrm{H}}$ is the absorption of the nuclear primary power-law, when the transmission scenario is employed to model the hard X-ray emission.

\begin{tabular}{lccccccc}
\hline \hline Scenario & $\Gamma$ & $\begin{array}{c}N_{\mathrm{H}} \\
\left(10^{23} \mathrm{~cm}^{-2}\right)\end{array}$ & $\begin{array}{c}f_{\mathrm{s}} \\
(\%)\end{array}$ & $\begin{array}{c}E_{\mathrm{c}} \\
(\mathrm{keV})\end{array}$ & $\begin{array}{c}I_{\mathrm{c}} \\
\left(10^{-5} \mathrm{~cm}^{-2} \mathrm{~s}^{-1}\right)\end{array}$ & $\begin{array}{c}E W \\
(\mathrm{eV})\end{array}$ & $\chi^{2} /$ d.o.f. \\
\hline Transmission & $1.8 \pm_{0.5}^{0.4}$ & $2.6 \pm_{0.8}^{1.7}$ & $10 \pm_{10}^{7}$ & $6.44 \pm_{0.06}^{0.07}$ & $4.9 \pm_{1.6}^{1.4}$ & 1160 & $90.0 / 101$ \\
Reflection-dominated $^{a}$ & $2.0 \pm_{0.6}^{0.5}$ & $\ldots$ & $\ldots$ & $6.43 \pm_{0.06}^{0.09}$ & $3.1 \pm_{0.9}^{1.1}$ & 1010 & $93.6 / 102$ \\
Reflection-dominated $^{b}$ & $2.0 \pm 0.5$ & $0.5 \pm_{0.4}^{0.7}$ & $\ldots$ & $6.44 \pm_{0.06}^{0.07}$ & $3.1 \pm 1.0$ & 880 & $87.8 / 101$ \\
\hline
\end{tabular}

${ }^{a}$ The reflection component is photoelectrically absorbed by $N_{\mathrm{H}, \text { Gal }}$ only.

${ }^{b}$ The reflection component is photoelectrically absorbed by a screen of column density $N_{\mathrm{H}}$.

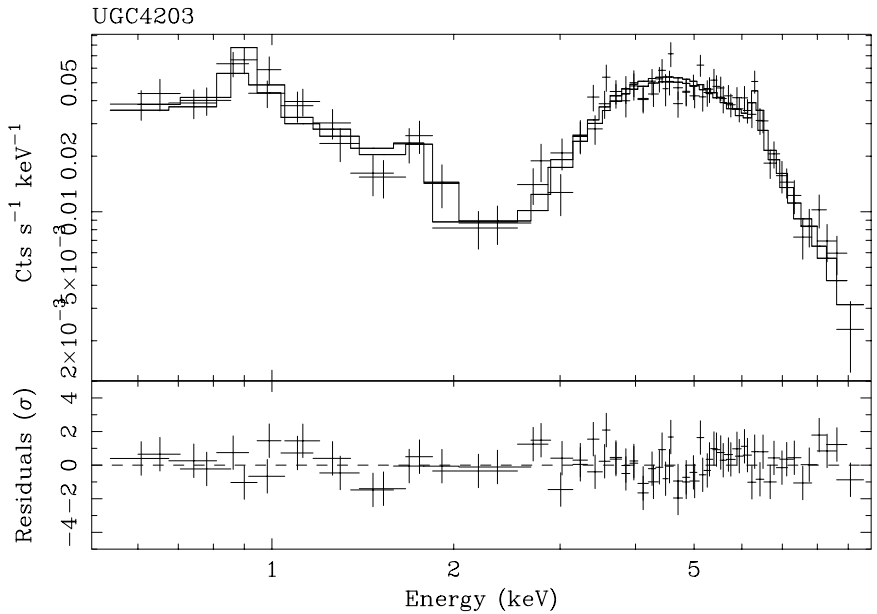

Fig. 2. UGC 4203 MOS spectra (upper panel) and residuals in units of standard deviations (lower panel) when the best-fit scattering scenario model is applied.

during the ASCA observation was significantly fainter than during the XMM-Newton one. The fluxes measured by ASCA are $2.3 \times 10^{-13} \mathrm{erg}^{\mathrm{cm}^{-2}} \mathrm{~s}^{-1}$, and $2.1 \times 10^{-12} \mathrm{erg} \mathrm{cm}^{-2} \mathrm{~s}^{-1}$ in the $0.5-2 \mathrm{keV}$ and $2-10 \mathrm{keV}$ energy band, respectively. We have first tried to fit the spectra with the same model adopted to fit the XMMNewton spectrum (transmission scenario in Table 2). Although the fit is acceptable $\left(\chi_{\nu}^{2}=0.89\right)$ there is no obvious explanation for the huge $(E W \simeq 1.2 \mathrm{keV})$ iron emission line (Leahy \& Creighton 1993; Matt 2002). This feature is instead naturally explained in the reflectiondominated scenario, appropriate for nuclei either absorbed by Compton-thick matter (Matt et al. 1996) or switched-off (Guainazzi et al. 1998), where emission lines are observed against the reflection continuum only (Matt et al. 1996). We have therefore substituted the absorbed hard X-ray power-law with an unabsorbed Compton-reflection component (model pexrav in XSPEC, Magdziarz \& Zdziarski 1995; reflection-dominated scenario in Table 2). We fixed the abundances to their solar values, and assumed reflection from an edge-on planeparallel slab. This model describes the data equally well $\left(\chi_{\nu}^{2}=0.90\right)$, but it is to be preferred on physical grounds. Even if the fit is already satisfactory, we have later included in the model an additional photoelectric absorption covering the Compton-reflection component, to verify whether the latter is also obscured by a Compton-thin absorber similar to the one found in the XMM-Newton observation. The the quality of the fit slightly improves $\left(\Delta \chi^{2}=5.8\right.$, corresponding to the $98.8 \%$ confidence level according to the $F$-test). This remains true also if the inclination angle and the abundances are left free to vary in the fit. The best-fit column density value is four times lower than, but still consistent at the $3 \sigma$ level (see Fig. 3) with that derived from the XMM-Newton spectrum.

The ASCA spectra do not provide significant constraints on the iron line profile or on the nature of the soft X-ray emission. Despite the remarkable flux change in the $2-10 \mathrm{keV}$ band between the XMM-Newton and the ASCA observations, the intensity of the iron line remained basically unchanged.

\section{Discussion}

The two X-ray observations of UGC 4203 described in this paper caught the source in two remarkably different spectral states. The ASCA spectrum is most-likely dominated by Compton-reflection. We assume in what follows that this component is due to reflection from the far inner side of Compton-thick matter surrounding the nucleus. We are therefore implicitly assuming an axisymmetric distribution of this matter, as in the torus envisaged in the 


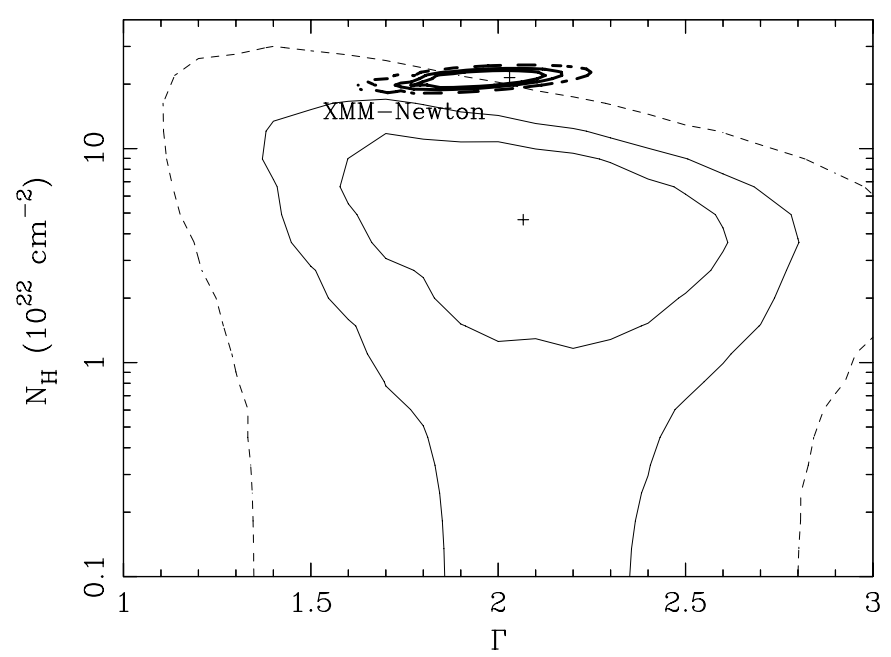

Fig. 3. Iso- $\chi^{2}$ contour plot for the intrinsic power-law spectral index versus the Compton-thin column density in the ASCA and XMM-Newton (small contours in bold) observation. In the former, the Compton-thin absorber covers the reflectioncomponent only. Contours are at $68 \%, 90 \%$ (solid lines) and 99\% (solid line) confidence level for two interesting parameters.

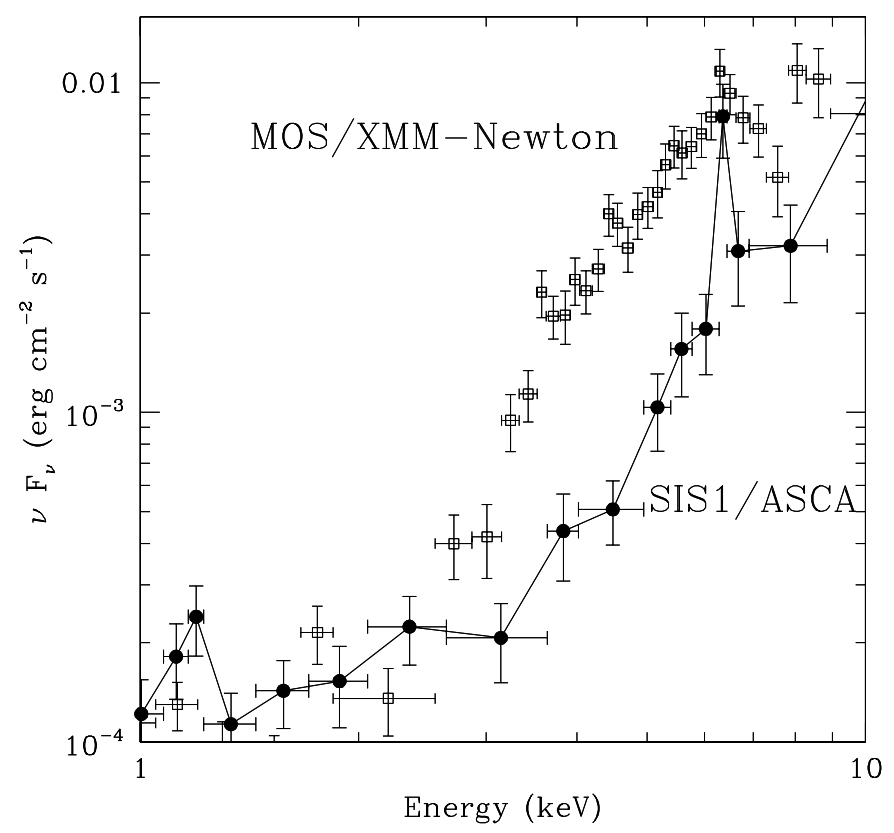

Fig. 4. X-ray spectral energy distribution of UGC 4203 during the ASCA (connected filled circles) and the XMM-Newton (empty squares) observations.

standard unification scenarios (Antonucci \& Miller 1985; Antonucci 1993). In the XMM-Newton observation of UGC 4203, however, the AGN was shining again (albeit through a Compton-thin absorber with $N_{\mathrm{H}} \simeq 2 \times$ $10^{23} \mathrm{~cm}^{-2}$ ). In Fig. 4 we compare the UGC $4203 \mathrm{X}$ ray spectral energy distributions measured by ASCA and XMM-Newton. Despite the $2-10 \mathrm{keV}$ flux difference, the intensities of the iron line in the two observations are consistent with each other. It is interesting to note that comparable fluxes are measured also in the soft X-rays, which are likely to be dominated by reprocessing or diffuse

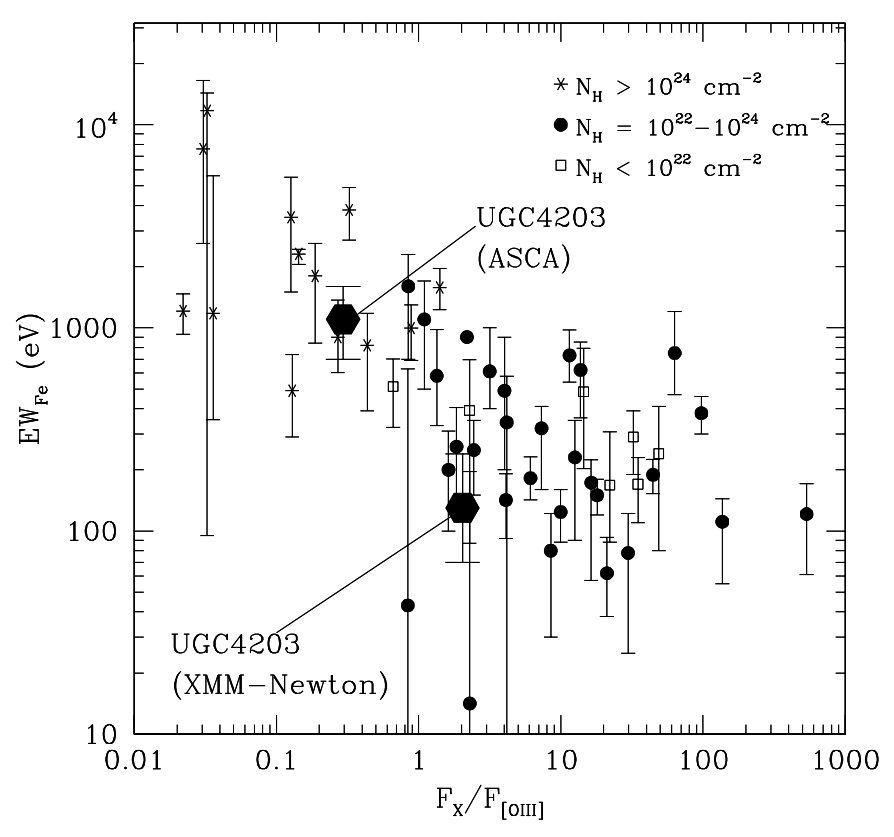

Fig. 5. "Bassani" diagnostic plot: $\mathrm{K}_{\alpha}$ fluorescent iron line $E W$ against the ration between the $2-10 \mathrm{keV}$ and the Balmerdecrement corrected OIII flux. The position of UGC 4203 in the ASCA and XMM-Newton observation is indicated by the big filled exagons.

emission. The lack of information about the history of the activity in UGC 4203 prevents us from deriving quantitative constraints on the location of the matter, which emits the bulk of the radiation at $\$ 2 \mathrm{keV}$.

In Fig. 5, the transition of UGC 4203 between a "Compton-thick" and a "Compton-thin" state is put in the context of the diagnostic diagram of Bassani et al. (1999). The iron line $E W$ is plotted against the ratio between the $2-10 \mathrm{keV}$ and the $\mathrm{O}[\mathrm{III}]$ flux corrected for the Balmer-decrement (taken from Ho et al. 1997). The path followed by UGC 4203 in this diagram is that expected from the above transition. Although variations of the X-ray absorber in Compton-thin objects (rarely larger than a factor of a few) are quite common (Risaliti et al. 2001), there are only two other convincing cases where a transition between a Comptonthin and -thick state can be claimed: NGC 1365; (Iyomoto et al. 1997; Risaliti et al. 2000), and NGC 6300 (Guainazzi 2001), out of about 30 objects for which at least two historical measurements of $N_{\mathrm{H}}$ are available.

The transition of UGC 4203 can be due to either: 1) a change in the properties of the absorber(s); 2) or a "switching-off" of the nucleus during the ASCA observation, leaving the Compton-reflection as the only "echo" of its previous higher activity level.

The timescale, on which the transition occurred, rules out an explanation in terms of "disappearance" of a single, homogeneous Compton-thick axis-symmetric system, covering a steady-state active nucleus. The covering fraction of the torus must be $\sim 2 \pi$ to account for the residual flux observed by ASCA. Large torus covering fractions are also 
suggested by statistical arguments (Maiolino et al. 1995; Risaliti et al. 2000). If the observed transition is related to the line-of-sight crossing time of a single spherical cloud in Keplerian motion around a $M_{8} \equiv M / 10^{8} M_{\odot}$ black hole, the distance from the central engine is $\sim 8 \times 10^{16} M_{8}^{1 / 3} \mathrm{~cm}$. A rather unlikely geometry of the torus would be necessary to explain the transition in this scenario.

However, an explanation along the same line of thought is viable, if the Compton-thick matter is clumpy. The crossing-time of a Keplerian cloud covering a region of size $r_{10}=r / 10 R_{\mathrm{S}}$ around the black hole is:

$t_{\mathrm{c}} \simeq 0.13 M_{8}^{1 / 2} d^{1 / 2} r_{10}$ years

where $d$ is the cloud distance from the nucleus in parsecs.

Alternatively, ASCA may have observed the AGN in UGC 4203 in a dim state. Compton-reflection would be therefore only the "echo" of its previous activity, later revived, and discovered by the XMM-Newton observation (the "Phoenix galaxy" effect). UGC 4203 would in this case join, e.g., NGC 4051 (Guainazzi et al. 1998; Uttley et al. 1999) and NGC 2992 (Gilli et al. 2000), whose dimming and subsequent recovery on timescales of the order of a fraction of year to a few years have been already monitored. In both cases the fainter states correspond to flat $\mathrm{X}$-ray spectra with strong $\mathrm{K}_{\alpha}$ fluorescent iron lines, interpreted as the emerging of reflection from Compton-thick matter at distances $\gtrsim 0.1 \mathrm{pc}$.

The implications of the latter explanation would be far-reaching. In the framework of the Seyfert unification scenario, X-ray reflection-dominated Seyfert 2 galaxies are strictly identified with Compton-thick objects. Recent hard X-ray studies (Salvati et al. 1997; Maiolino et al. 1998; Risaliti et al. 2000) suggest that they may constitute about half of the Seyfert 2 population, at least in the local Universe. If, on the other hand, reflection-dominated states are due to AGN low-activity phases, their rate of occurrence may be telling us more on the duty-cycle of the AGN phenomenon, rather than on the geometry of the nuclear environment.

It is admittedly difficult to discriminate between the two above scenarios, given the paucity of information on the X-ray history of UGC 4203. In NGC 6300 (Guainazzi 2001), a similar transition from a reflection- to a transmission-dominated status was observed. However, a remarkably strong residual Compton-reflection component was clearly present in the latter state, a factor of 4 higher than typically observed in unabsorbed Seyfert galaxies. Since in the latter the reflector covers probably a $\approx \pi$ solid angle to the black hole, the most straightforward explanation for this finding is to invoke an intrinsic change of the AGN primary emission, probably larger than an order of magnitude. NGC 6300 is the best demonstration to date that the reflection- to transmission-dominated transitions are indeed related to variation of the AGN intrinsic luminosity. Unfortunately, the limited energy band of the EPIC cameras prevents us from verifying this hypothesis in the UGC 4203 case.

\subsection{Where is/are the absorber/s?}

Independently of which of the above explanations is correct, the results presented in this paper have implications on the nature and/or location of the matter responsible for the X-ray photoelectric absorption in Seyfert 2 galaxies.

A "clumpy torus" may explain the observed transition in UGC 4203. We may be observing UGC 4203 through the unstable or uneven torus rim, causing clouds of different column densities to appear along the line-of-sight to the nucleus due to local instabilities or AGN-driven clouds evaporation (Pier \& Voit 1995). Clumpy torii may be thermally and dynamically supported by the AGN radiation field, as shown by Pier \& Krolik (1992). Magnetic fields may play a role as well. Although more theoretical work and fully self-consistent calculations are needed, this seems a rather promising possibility to explain our results.

If, on the other hand, the transition is due to a changed AGN activity, it follows that the Compton-thin absorbing matter may be different than the torus, whose presence in UGC 4203 is implied by the reflection-dominated, X-ray dim state observed by ASCA. A possible location of this additional absorber was suggested by Matt (2000). In his extension of the traditional unification scenario, the compact, dusty torus is still responsible for the Compton-thick $\mathrm{X}$-ray nuclear absorption only, whereas the Compton-thin absorbers are located at much larger distances. If this scenario applies to UGC 4203 as well, the Compton spectrum in the reflection-dominated state should be absorbed by Compton-thin matter. The quality of the ASCA spectrum is unfortunately not good enough to give a definitive answer to this question. However, an additional Comptonthin absorber is required at the $98.8 \%$ level in the ASCA reflection-dominated state.

Alternatively, BLR clouds are expected to have a column density of $N_{\mathrm{H}} \approx 10^{23} \mathrm{~cm}^{-2}$ as well. However, the possibility that they are responsible for the X-ray Comptonthin absorption is ruled out by the low covering fraction of the BLR as estimated from the lack of Ly-edge cutoff or carbon dip in the UV spectrum of type 1 AGN (Maiolino et al. 2001) and the lack of silicate absorption feature in the ISO spectra of AGN (Clavel et al. 2000).

\section{Summary}

Comparing an autumn 1995 ASCA with a summer 2001 XMM-Newton observation of the Seyfert 2 galaxy UGC 4203 (Mkn 1210), we discovered a rare transition from a Compton reflection- to a transmission-dominated state. This transition can be explained by one of the following scenarios:

- The "disappearance" of a Compton-thick cloud, covering a steady-state active nucleus at the time of the ASCA observation. If this explanation is correct, the outcomes presented in this paper suggest that the compact molecular torus, encompassing the nuclear environment in the Seyfert unification scenarios, has actually a clumpy or patchy structure. 
- A transition in the activity level of the UGC 4203 AGN. The XMM-Newton would have unveiled its revival after the "silent" phase caught by ASCA (the "Phoenix galaxy" effect).

The latter explanation would imply that, at least in this object (and probably in the objects where this kind of transitions occurs (Guainazzi 2001)) the Compton-thin and Compton-thick absorbers are different. This would provide support to models where the standard "torus" intercepts the line of sight to the nucleus in Comptonthick objects only (Matt 2000), whereas Compton-thin absorbers are located at larger scales, maybe associated with the host galaxy rather then with the nuclear environment.

Acknowledgements. Constructive comments from an anonymous referee, which greatly improved the quality of the presentation, are gratefully acknowledged. The authors acknowledge as well helpful discussions with J. Krolik on the radiation-supported torii models, and stimulating historical remarks by R. R. J. Antonucci. FF, GCP and GM acknowledge financial support from ASI and the Italian Ministry of Research, under grant COFIN-00-02-36.

\section{References}

Antonucci, R. R. J. 1993, ARA\&A, 31, 473

Antonucci, R. R. J., \& Barvainis, R. 1990, ApJ, 367, L17

Antonucci, R. R. J., \& Miller, J. S. 1985, ApJ, 297, 621

Awaki, H., Koyama, K., Inoue, H., \& Halpern, J. O. 1991, PASJ, 43, 195

Awaki, H., Ueno, S., Taniguchi, Y., \& Weaver, K. A. 2000, ApJ, 542, 175

Bassani, L., Dadina, M., Maiolino, R., et al. 1999, ApJS, 121, 473

Bianchi, S., Matt, G., \& Iwasawa, K. 2000, MNRAS, 322, 669

Braatz, J. A., Wilson, A. S., \& Henkel, C. 1994, ApJ, 437, L99 Clavel, J., Schultz, B., Altieri, B., et al. 2000, A\&A, 357, 839

Dickey, J. M., \& Lockman, F. J. 1990, ARA\&A, 28, 215

Gilli, R., Maiolino, R., Marconi, A., et al. 2000, A\&A, 355, 485

Guainazzi, M. 2001, MNRAS, 329, L13
Guainazzi, M., Fiore, F., Matt, G., \& Perola, G. C. 2001, MNRAS, 327, 323

Guainazzi, M., Nicastro, F., Fiore, F., et al. 1998, MNRAS, 301, L1

Ho, L. C., Filippenko, A. V., \& Sargent, W. L. W. 1997, ApJS, 112,315

Keel, W. C. 1980, AJ, 85, 198

Iyomoto, N., Makishima, K., Fukazawa, Y., Tashiro, M., \& Ishisaki, Y. 1997, PASJ, 49, 425

Jansen, F., Lumb, D., Altieri, B., et al., A\&A, 365, L1

Leahy, D. A., \& Creighton, J. 1993, MNRAS, 263, 314

Magdziarz, P., \& Zdziarski, A. A. 1995, MNRAS, 273, 837

Maiolino, R., Marconi, A., Salvati, M., et al. 2001, A\&A, 365, 28

Maiolino, R., Ruiz, M., Rieke, G. H., \& Keller, L. D. 1995, ApJ, 446, 561

Maiolino, R., Salvati, M., Bassani, L., et al. 1998, A\&A, 338, 781

Malkan, M. A., Gorijn, V., \& Tam, R. 1998, ApJS, 117, 25

Matt, G. 2000, A\&A, 355, L13

Matt, G., Brandt, W. N., \& Fabian, A. C. 1996, MNRAS, 280, 823

Matt, G. 2002, MNRAS, submitted

Peterson, B. 1993, PASP, 105, 247

Pier, E. A., \& Krolik, J. H. 1992, ApJ, 399, L23

Pier, E. A., \& Voit, G. M. 1995, ApJ, 450, 628

Risaliti, G., Elvis, M., \& Nicastro, F. 2001, ApJ, in press [astro-ph/0107510]

Risaliti, G., Maiolino, R., \& Bassani, L. 2000, A\&A, 356, 33

Sako, M., Kahn, S. M., Paerels, F., \& Liedhal, D. A. 2000, ApJ, 543, L115

Salvati, M., Bassani, L., della Ceca, R., et al. 1997, A\&A, 323, L1

Sambruna, R., Netzer, H., Kaspi, S., et al. 2001, 546, L13

Strüder, L., Briel, U., Dannerl, K., et al. 2001, A\&A, 365, L18

Tran, H. D. 1995, ApJ, 440, 565

Turner, T. J., George, I. M., Nandra, K., \& Mushotzky, R. F. 1997, ApJS, 113, 23

Turner, M. J. L., Abbey, A., Arnaud, M., et al. 2001, A\&A, 365, L27

Uttley, P., McHardy, I., Papadakis, I. E., Guainazzi, M., \& Fruscione, A. 1999, MNRAS, 307, L6 